University of Nebraska - Lincoln

DigitalCommons@University of Nebraska - Lincoln

Faculty Publications from the Department of Engineering Mechanics

$10-1-2003$

\title{
Method for Performing Accelerated Characterization of Viscoelastic Constitutive Behavior of Asphaltic Concrete
}

Curtis Berthelot

University of Saskatchewan

D. H. Allen

University of Nebraska-Lincoln, dallen3@unl.edu

Chad Searcy

Texas, A\&M University, College Station

Follow this and additional works at: https://digitalcommons.unl.edu/engineeringmechanicsfacpub

Part of the Mechanical Engineering Commons

Berthelot, Curtis; Allen, D. H.; and Searcy, Chad, "Method for Performing Accelerated Characterization of Viscoelastic Constitutive Behavior of Asphaltic Concrete" (2003). Faculty Publications from the Department of Engineering Mechanics. 49.

https://digitalcommons.unl.edu/engineeringmechanicsfacpub/49

This Article is brought to you for free and open access by the Mechanical \& Materials Engineering, Department of at DigitalCommons@University of Nebraska - Lincoln. It has been accepted for inclusion in Faculty Publications from the Department of Engineering Mechanics by an authorized administrator of DigitalCommons@University of Nebraska - Lincoln. 


\title{
Method for Performing Accelerated Characterization of Viscoelastic Constitutive Behavior of Asphaltic Concrete
}

\author{
Curtis F. Berthelot, ${ }^{1}$ David H. Allen, ${ }^{2}$ and Chad R. Searcy ${ }^{3}$ \\ 1 Assistant Professor, Department of Civil Engineering, University of Saskatchewan, 57 Campus Drive, Saskatoon SK, Canada S7N5A9. \\ 2 Professor, Department of Aerospace Engineering, Texas A\&M University, College Station, TX 77843-3141. \\ 3 Graduate Student, Department of Aerospace Engineering, Texas, A\&M University, College Station, TX 77843-3141.
}

\begin{abstract}
Constitutive models based in continuum mechanics are formulated for modeling the time dependent mechanical response of asphaltic concrete. The model chosen for the initial phase of study is linear viscoelastic, resulting in stress-strain relations that are of the single integral Boltzmann type in time. Experimental equipment and testing protocols are described for obtaining material properties within the viscoelastic framework selected for characterization. A frequency sweep experimental technique is described for obtaining composite properties from short-term tests that can be used to predict long-term, time-dependent material behavior. Mathematical techniques are described for converting experimentally obtained complex compliances to creep compliances and relaxation moduli in the time domain. These techniques are then used to produce the relaxation moduli for four different mixes of asphaltic pavement.
\end{abstract}

Keywords: asphaltic concrete, constitutive models, viscoelasticity, asphalt pavements, mechanical properties

\section{Introduction}

Asphaltic pavement is a primary surface coating used in the Canadian province of Saskatchewan. Recent governmental deregulation of the Canadian railway system has accelerated trucking traffic on these roadways, thereby increasing the failure rate of the Saskatchewan roadway system. Existing empirical techniques for designing these roadways have been unsuccessful in avoiding premature failures of roadways. Indeed, several roadways have failed within 3 years of their inception. As a means of improving the design methodology so that premature failures can be mitigated, models are currently under development that are based in continuum mechanics for predicting asphaltic pavement life as a function of the input design parameters associated with loads, geometry, and material properties. This is a formidable task due in particular to the myriad of design variables associated with material properties because the asphaltic mix can contain as many as seven different constituents. Additional design variables associated with geometry include the thicknesses of the various sub-grade coatings in addition to the thickness of the surface coating of asphalt. Furthermore, the prediction of life can be complicated due to the fact that there are at least three commonly encountered failure modes: surface rutting caused by excessive deformations, fatigue fracture, and thermal cracking.

The goal of this research effort is to develop a model that is capable of predicting failure of asphaltic pavement by all of these modes. The solution to this problem requires that at least three different problems be addressed: (1) the prediction of thermomechanical constitutive behavior of asphalt concrete; (2) prediction of time-dependent response of associated asphaltic roadways; and (3) development of a physically based methodology for predicting failure of the roadway. Problems 2 and 3 are being addressed in other efforts by the authors and will be the subject of future articles. The current paper presents a combined theoretical and experimental methodology for developing mechanical constitutive models for asphaltic concrete.

\section{Recent Research on Asphaltic Pavement}

The design of asphalt roadways in Saskatchewan is currently performed using empirical models. Among these are the Hveem stability test (AASHTO 1995); ASTM 1996, the Marshall stability and flow test (White 1985; Foster 1993), the unconfined compressive strength test (AASHTO 1995); ASTM 1996, static and repeated load creep testing (Little et al. 1992), and flexural beam fatigue testing (Matthews and Monismith 1994). A description of each of these models can be found in work by Berthelot (1999). These models are based primarily on judgment inferred from past road performance observations. This approach involves assuming the performance predictor variables and quantifying the predictor coefficients based on regression analysis of statistical data sets obtained from past roadway observations. Once formulated, a model is evaluated for accuracy and statistical significance, and the statistically insignificant variables are removed from the model. Therefore, it is required that full-scale road experiments be performed for all of the test variables involved. Clearly, this approach is at the very least quite costly. Furthermore, when new materials become available, which is often the case, the experimental program must be expanded. In addition, no insight is gained from such empirical approaches as to how design approaches might be improved. Finally, the existing preponderance of roadway failures gives ample evidence of the failure of these models to perform effectively. Thus, more physically based models are needed in order to mitigate costly roadway failures in the future. 
In the past decade, considerable research has been performed to construct physically based models for asphaltic pavement. No attempt will be made to cover the entire body of research on this subject. Instead, a short review of those approaches that treat the pavement as viscoelastic will be discussed. Indeed, most of these appear to fall into one distinct approach in that they are based on a formulation proposed by Schapery for particulate composites (Schapery 1981; 1982, 1984a, 1987a,b, 1990a,b, 1994; Schapery and Sicking 1995). Briefly, this approach uses a single integral framework in time to account for history and rate dependence, together with a nonlinear strain scale, to construct a nonlinear viscoelastic stress-strain relation at the composite level for asphaltic concrete. As such, the effects of microcracking in both binder and aggregate are accounted for phenomenologically in the nonlinear strain scale. Such an approach is often termed "continuum damage mechanics" because the microcracks are not treated explicitly as time-dependent internal boundaries. Rather, the average dissipation caused by these microcracks is accounted for at the mesoscale of the composite by including irreversibility phenomenologically. Several researchers have characterized the behavior of asphaltic binder using this approach (Kim and Little 1990; Kim et al. 1995; Park et al. 1996; Lee et al. 2000).

Although the previous recent research efforts are quite advanced and as such represent the current state-of-the-art in characterization of the constitutive behavior of asphaltic pavement, they do not cogently address two significant physical features of asphaltic concrete. First, these models do not perform an analysis on the microscale. That is, they do not account for the geometric and material effects at the level of asphalt binder and aggregate, including microcracking. The authors have addressed this issue in another paper on this subject (Allen et al. 2001b). Second, they do not use accelerated testing protocols, so that in order to predict long-term thermoviscoelastic response, long-term experiments must be performed.

A second approach has been reported recently for predicting the viscoelastic response of asphaltic concrete (Kim et al. 1997). While this approach does not include the effect of microcracking, it does use an accelerated testing technique. The authors of the current paper have developed a new experimental technique for accelerated testing that is substantially different from that previously reported (Kim et al. 1997), and this will be developed herein.

\section{Viscoelastic Material Model}

In this section, a general approach to modeling thermoviscoelastic constitutive behavior of asphalt concrete will be formulated. This will be cast within the framework of continuum mechanics (Truesdell and Noll 1965; Malvern 1969).

Thermoviscoelastic materials exhibit rate and history dependence. This is reflected in the thermomechanical constitutive equations relating the kinetic variables to the kinematic variables. Asphalt shows this type of dependence, as can be demonstrated readily by hanging an object of constant mass from a specimen of the material and observing that the deformations are time dependent. For example, if the strain is used as the input variable, a strain formulation is obtained as follows:

$$
\sigma_{i j}(\vec{x}, t)=\Omega_{\tau=-\infty}^{\tau=t}\left\{\varepsilon_{k l}(\vec{x}, \tau), T(\vec{x}, \tau)\right\}
$$

where $\sigma_{i j}=$ the stress tensor; $\varepsilon_{k l}=$ the strain tensor; $T=$ the temperature; $\tau$ is the generic timescale; $\overrightarrow{\mathbf{x}}=$ the coordinate location of interest, and $t=$ the current time of interest; Thus, $\Omega\{\}$ de- notes a functional mapping of the entire past history of strain and temperature into the current stress state at time $t$. Because only strain and temperature are included in the functional, this is called a simple material (Truesdell and Noll 1965). An inverse formulation, called a stress formulation, is given by

$$
\varepsilon_{i j}(\vec{x}, t)=\Theta_{\tau=-\infty}^{\tau=t}\left\{\sigma_{k l}(\vec{x}, \tau), T(\vec{x}, \tau)\right\}
$$

where, presumably, the mapping between $\Omega\{\}$ and $\Theta\{\}$ is bijective (one-to-one and onto) (Oden 1979). A variety of materials exhibit linear thermoviscoelastic material behavior, so that either Equation (1) or Equation (2) can be expanded linearly in convolution integrals in the functional arguments. Thus, expanding Equation (1) (for the nonaging case) results in

$$
\sigma_{i j}(t)=\int_{\tau=-\infty}^{\tau=t} E_{i j k l}(t-\tau) \frac{\partial \varepsilon_{k l}}{\partial \tau} d \tau+\int_{\tau=-\infty}^{\tau=t} \beta_{i j}(t-\tau) \frac{\partial T}{\partial \tau} d \tau
$$

where $E_{i j k l}(\tau)$ is called the relaxation modulus tensor, $\beta_{i j}(\tau)$ is called the thermal relaxation expansion tensor, and the positional notation has been excluded for simplicity. These two tensors constitute the material properties that must be characterized should the material be linear. Alternatively, Equation (2) may be expanded to obtain a linear stress formulation as follows:

$$
\varepsilon_{i j}(t)=\int_{\tau=-\infty}^{\tau=t} D_{i j k l}(t-\tau) \frac{\partial \sigma_{k l}}{\partial \tau} d \tau+\int_{\tau=-\infty}^{\tau=t} \alpha_{i j}(t-\tau) \frac{\partial T}{\partial \tau} d \tau
$$

where $D_{i j k l}(\tau)$ is called the creep compliance tensor and $\alpha_{i j}(\tau)$ is called the thermal compliance expansion tensor. In the current paper only, isothermal mechanical response will considered, so that the terms involving temperature may be neglected in Equations (1)-(4). Although thermal effects are being studied by the authors (Berthelot 1999), this will be the subject of a future paper.

As will be shown below, there exist transformation formulas relating the relaxation moduli in Equation (3) to the creep compliances in Equation (4). Thus, in principal it is not necessary to obtain both sets of material properties experimentally. From a practical standpoint, it is generally less cumbersome to characterize the creep compliances. To see why this is the case, consider an isothermal experiment with applied stresses that are constant in time (called a creep test). The time derivatives in the first integral term in Equation (4) will produce Dirac delta functions (Greenburg 1978), so that integration of Equation (4) will result in

$$
\varepsilon_{i j}(t)=D_{i j k l}(t) \sigma_{k l}^{0}
$$

where, by definition

$$
\sigma_{k l}^{0} \equiv \sigma_{k l}\left(t=0^{+}\right)
$$

Thus, Equation (5) can be inverted to obtain

$$
D_{i j k l}(t)=\varepsilon_{i j}(t) / \sigma_{k l}^{0}
$$

During the experiment, the following additional equations must hold:

(1) Conservation of linear momentum

$$
\frac{\partial \sigma_{j i}}{\partial x_{j}}=0 \quad \text { in } V
$$

where we have assumed that body forces and inertial effects are negligible during the experiment, and on the boundary

$$
T_{i}=\sigma_{j i} n_{j} \quad \text { on } \partial V
$$


where $\partial V$ is the boundary of the body with volume $V$, and $T_{i}$ is the traction vector (Malvern 1969).

(2) Conservation of angular momentum

$$
\sigma_{j i}=\sigma_{i j} \text { in } V+\partial V
$$

assuming that there are no body moments.

(3) Strain-displacement relations

$$
\varepsilon_{i j}=\frac{1}{2}\left(\frac{\partial u_{i}}{\partial x_{j}}+\frac{\partial u_{j}}{\partial x_{i}}\right) \text { in } V
$$

where $u_{i}=$ the displacement vector, and it is assumed that the displacement gradients are small during the experiment (Malvern 1969). In addition, the conservation of energy must be satisfied during the experiment for the case where transient temperatures are considered. However, for simplicity, this circumstance will not be considered in the present paper.

Now, constitutive Equations (3) or (4), together with field Equations (8)-(10), may be adjoined with appropriate initial and boundary conditions to produce a model capable of predicting the stresses, strains, and displacements as functions of space and time for an object of arbitrary shape, so long as the material properties in Equations (3) or (4) are known a priori. Furthermore, failure may be predicted by a variety of mechanisms such as fracture, so long as a failure criterion can be constructed as a function of these variables, such as the Griffith criterion for fracture (Griffith 1920). Therefore, completing the model description requires that the material properties in Equations (3) or (4) be evaluated. This may be accomplished as follows.

Consider an experiment in which stresses are spatially homogeneous. We will term this a constitutive experiment. Then, for this circumstance, conservation of linear momentum Equation $(8 a)$ is trivially satisfied within the body. Furthermore, the stresses within the body can be determined by using Equation $(8 b)$, so long as the tractions are known on the boundary of the body. These can be obtained from a load cell or a similar device. In addition, from Equation (4) it can be seen that if the stresses are spatially homogeneous, then the strains are also spatially homogeneous in the body. Thus, strain-displacement Equation (10) can be integrated in spatial coordinates to obtain the values of the strain, given the boundary displacements (obtained from linear voltage differential transducers (LVDTs). Thus, it is apparent that in a constitutive test the values on the right hand side of Equation (7) may be determined, so that the creep compliance $D_{i j k l}(t)$ may be evaluated from this experiment.

Of course, asphaltic roadways subjected to vehicular traffic and environmental conditions do not undergo spatially homogeneous stress states. However, the attractive feature of the continuum mechanics formulation outlined previously is that the material properties obtained from the constitutive experiments are independent of the geometry of the problem to be solved. The values obtained for $D_{i j k l}(t)$ may then be utilized in conjunction with the conservation of linear momentum Equations (8) and (9), the conservation of energy, and the straindisplacement Equation (10) to construct a well-posed initial boundary value problem (IBVP) for a roadway of arbitrary geometry. The solution of the IBVP by analytic or computational means will result in predictions of the stresses, strains, and displacements as functions of position and time in an asphaltic roadway due to environment and mechanical loading (Truesdell and Noll 1965; Malvern 1969). Accurate predictions of the stresses, strains, and displacements can be used to predict failure by a variety of modes, such as fracture. These modeling results can then be used as a tool for designing asphaltic roadways with improved life, by sweeping out the design variables associated with loading, pavement geometry, and material properties of the asphaltic concrete and subbase.

The second author and coworkers at Texas A\&M University have developed a finite-element algorithm that can be used to predict the response of thermoviscoelastic asphaltic roadways to arbitrary quasi-static mechanical and thermal loading, including cyclic loadings (Allen et al. 1994; Zocher et al. 1997). Therefore, the characterization of the material properties described above constitutes a significant impediment to modeling the predicted response of an asphaltic roadway. The following section will describe an experimental procedure for obtaining these properties.

\section{Experimental Techniques}

In the case wherein the material is isotropic, it can be shown that the creep compliance reduces to the following form (Christensen 1982):

$$
D_{i j k l}(t)=\frac{1}{3}\left[J_{2}(t)-J_{1}(t)\right] \delta_{i j} \delta_{k l}+\frac{1}{2} J_{1}(t)\left[\delta_{i k} \delta_{j l}+\delta_{i l} \delta_{j k}\right]
$$

where $\delta_{i j}$ is the Kroneker delta (Malvern 1969). Thus, there are only two independent components of the creep compliance that must be determined experimentally for an isotropic linear viscoelastic material. While it is entirely reasonable to find out that the deposition process will lead to some anisotropy in asphalt roadways, it will be assumed in the initial phase of research that asphalt is macroscopically isotropic. By macroscopically isotropic, we imply that specimens will be tested that contain a large number of randomly oriented and sized reinforcement particles, so that the material is statistically homogeneous, that is, standard deviations of aggregate size and shape are small compared to their means. Therefore, the immediate objective of the research is to determine $J_{1}(t)$ and $J_{2}(t)$ for asphalt. This in itself is not an easy task due to the complicated nature of asphalt. In order to assign physical significance to the two isotropic compliances described in Equation (11), suppose we introduce the deviatoric stress and strain tensors, defined by

$$
\sigma_{i j}^{\prime} \equiv \sigma_{i j}-\frac{\sigma_{k k}}{3} \delta_{i j}
$$

and

$$
\varepsilon_{i j}^{\prime} \equiv \varepsilon_{i j}-\frac{\varepsilon_{k k}}{3} \delta_{i j}
$$

Thus, substituting Equation (11) and the previous definitions into constitutive Equation (4) results in (for isothermal conditions.

and

$$
\varepsilon_{k k}(t)=\int_{-\infty}^{t} J_{2}(t-\tau) \frac{\partial \sigma_{k k}}{\partial \tau} d \tau
$$

$$
\varepsilon_{i j}^{\prime}(t)=\int_{-\infty}^{t} J_{1}(t-\tau) \frac{\partial \sigma_{i j}^{\prime}}{\partial \tau} d \tau
$$

Thus, for obvious reasons, $J_{1}(t)$ is often called the shear creep compliance, and $J_{2}(t)$ is called the bulk creep compliance.

An experimental testing procedure has recently been developed for obtaining these two compliances. In this test, a cylindrical specimen is subjected to boundary tractions (in load control. that lead to the following spatially homogeneous stress state:

$$
\sigma_{11}(t)=-p H(t)+\sigma^{0} e^{i \omega t}
$$




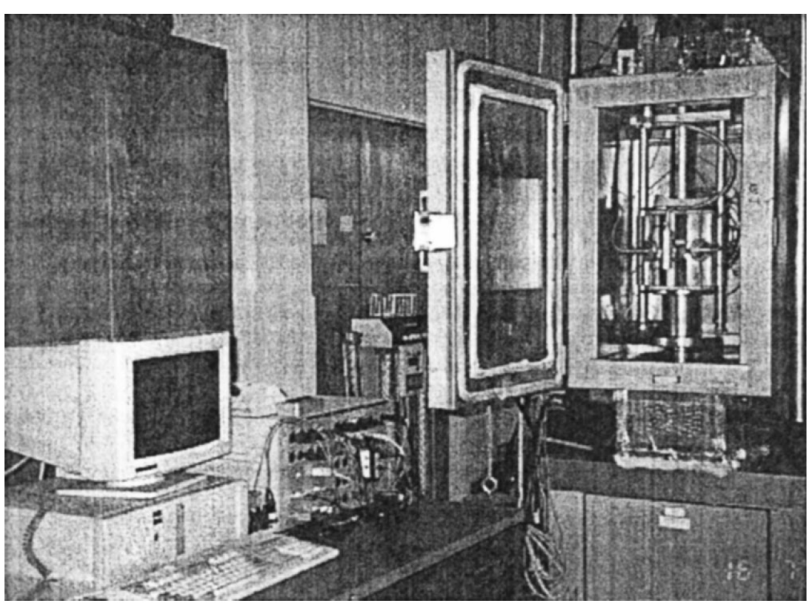

Figure 1. Photo of experimental configuration

where $x_{1}=$ the axial coordinate direction, and $H(t)=$ the Heaviside step function (Greenburg 1978), as well as

$$
\sigma_{22}(t)=\sigma_{33}(t)=-p H(t)
$$

where $\omega=$ frequency of the applied loading in the $x_{1}$ coordinate direction. All of the components of shearing stress $\left(\sigma_{23}\right.$, $\sigma_{13}, \sigma_{12}$ ) are zero on the planes normal to the coordinate axes in this experiment. The lateral pressure $p$ and axial stress amplitude $\sigma_{0}$ are held constant during the test, and the axial loading frequency $\omega$ is then swept out over the range at which variations in the specimen strains are observed.

This type of constitutive test is significantly simpler to perform on asphalt than uniaxial tensile tests, because of the inability of the binder to carry large tensile stresses. Furthermore, as will be shown later, cyclic loading tests can potentially be used to obtain accelerated long-term compliance with short- term experiments by sweeping out a range of different frequencies. In this work, we consider only loadings that produce no damage, so that the loads must necessarily be small. In the case where damage is induced during the experiment, another approach is required (Allen and Yoon 1998).

Triaxial testing has been used by the road industry to characterize all types of road materials (Texas 1995; Witczak and Fonseca 1996; Kim et al. 1997). However, there are drawbacks to the traditional geotechnical and Texas triaxial test apparati, such as limited test temperature testing capabilities, time consuming sample preparation and instrumentation, limited feedback control capabilities; limited dynamic testing capabilities, and difficult measurement of Poisson's ratio. To overcome these drawbacks, this research has employed a new prototype full-feedback controlled Rapid Triaxial Test (RATT) apparatus (Figures 1 and 2) to characterize the specimens studied herein. One of the benefits of the RATT over conventional triaxial test apparati is the ability to characterize samples prepared in the SHRP gyratory compactor without the need to saw-cut or trim the test sample. This ability greatly expedites sample characterization and, therefore, facilitates mechanistic characterization of asphalt concrete mixes on a production scale.

The RATT cell consists of a cylindrical chamber with an aspect ratio of 1:1 to accommodate standard gyratory compacted samples. The RATT cell employs a pneumatic confinement chamber with four linear variable differential transducers mounted axially around the sample. Axial tractions are applied by a standard servo-hydraulic test frame with two LVDTs mounted axially to measure vertical displacement. The RATT cell can be operated in load or displacement control where the radial and axial loads or displacements are controlled independently. Further description of this testing procedure can be found in work by Berthelot (1999).

The following two quantities are measured as a function of the loading frequency during the test:

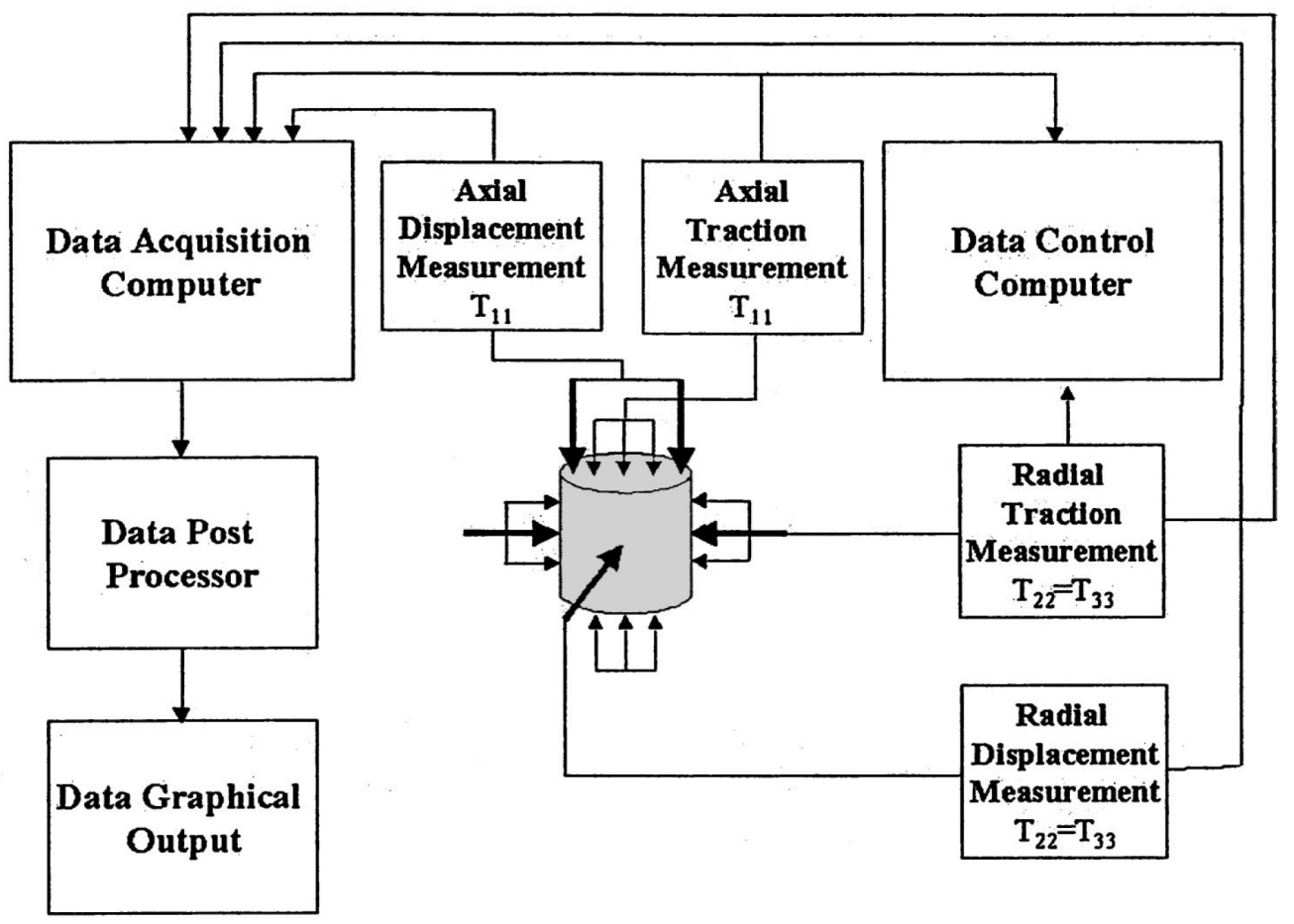

Figure 2. Schematic of experimental configuration 


$$
\alpha \equiv \frac{\varepsilon_{11}}{\sigma_{11}}
$$

and

$$
\beta \equiv \frac{-\varepsilon_{22}}{\varepsilon_{11}}
$$

Although the above two quantities are similar to the familiar Young's modulus and Poisson's ratio, respectively, they are not the same here because the loading is not uniaxial.

By direct substitution of Equations (16) and (17) into the isotropic form of Equation (4), it can be shown that during the test

$$
\varepsilon_{11}=\frac{2 J_{1}^{*}}{3} \sigma_{0} e^{i \omega t}+J_{2}^{*}\left(-p+\frac{\sigma_{0}}{3} e^{i \omega t}\right)
$$

and

$$
\varepsilon_{22}=\frac{J_{1}^{*}}{3} \sigma_{0} e^{i \omega t}+J_{2}^{*}\left(-p+\frac{\sigma_{0}}{3} e^{i \omega t}\right)
$$

Note that Equation (21) may also be written as follows:

$$
\varepsilon_{22}=\varepsilon_{11}-\frac{J_{1}^{*}}{3} \sigma_{0} e^{i \omega t}
$$

Thus, substituting Equations (20) and (22) into Equation (19) and rearranging this result gives

$$
J_{1}^{*}(\omega)=3(1+\beta) \frac{\varepsilon_{11}}{\left(\sigma_{11}+p\right)}
$$

where $J_{1}^{*}(\omega)$, called the complex shear compliance, is given by

$$
J_{1}^{*}(\omega) \equiv J_{1}^{\prime}(\omega)+i J_{1}^{\prime}(\omega)
$$

where

$$
J_{1}^{\prime}(\omega) \equiv J_{1}(\infty)+\omega \int_{0}^{\infty} J_{1}(\tau) \sin \omega \tau d \tau
$$

is called the shear storage compliance, and

$$
J_{1}^{\prime}(\omega) \equiv \omega \int_{0}^{\infty} J_{1}(\tau) \cos \omega \tau d \tau
$$

is called the shear loss compliance (Christensen 1982). Furthermore, substituting Equations (16) and (20) into Equation (18) results in

$$
\alpha=\frac{2 J_{1}^{*}}{3 \sigma_{11}} \sigma_{0} e^{i \omega t}+\frac{J_{2}^{*}}{\sigma_{11}}\left(-p+\sigma_{0} e^{i \omega t}\right)
$$

Substituting Equation (23) into the above and solving for $J_{2}^{*}$ gives

$$
J_{2}^{*}(\omega)=\alpha[1-2(1+\beta)] \frac{3 \sigma_{11}}{\left(-2 p+\sigma_{11}\right)}
$$

where, $J_{2}^{*}(\omega)$, called the complex bulk compliance, is defined similar to the definition given in Equations (24)-(26).

In order for the techniques described above to be appropriate for the materials considered, the materials must be linear viscoelastic, as described by the linear expansions in
Table 1. Mix Design Aggregate Proportions for Four Specimens Tested

\begin{tabular}{lrr}
\hline Aggregate type & SHT Marshall (\%) & Superpave (\%) \\
\hline Crushed coarse proportion & 33.6 & 64.3 \\
Crushed fines proportion & 13.9 & 14.9 \\
Natural fines proportion & 51.5 & 19.8 \\
Blender sand proportion & 0.0 & 0.0 \\
Reclaim asphalt concrete proportion & 0.0 & 0.0 \\
Lime proportion & 1.0 & 1.0 \\
\hline
\end{tabular}

Equations (3) or (4). The accuracy of the linearity assumption may be assessed for a particular material by checking the principles of homogeneity and superposition (Greenburg 1978). Homogeneity is satisfied if the viscoelastic moduli or compliances (in either the time domain or the frequency domain) are independent of the imposed loading level (or imposed displacement level) used during the test. The principle of superposition, which states that the responses to separate loadings may be superposed to obtain the response to the sum of the loadings, is most easily satisfied by performing so-called creep and recovery tests (Berthelot 1999). Generally, most materials are linear below a certain peak level of applied stress. However, beyond this level they will become nonlinear. In the case of asphaltic concrete, nonlinearity may be introduced by a variety of physical mechanisms, such as liquid mass diffusion, chemical changes, and microscale cracking. In the current paper, the authors have limited tests to stress levels in which these sources of nonlinearity are not observed, so that the experiments have demonstrated that the materials are linear for the stress levels considered herein (Berthelot 1999).

Experiments have been performed at $20^{\circ} \mathrm{C}$ on three specimens each of four different asphalt concrete mixes, with design aggregate proportions described in Table 1 . The first of these is categorized as an SHT Marshall mix, and the last three are Superpave mixes (Berthelot 1999). Aggregate size distributions for the four specimens are shown in Tables 2 and 3.

The four mixes were tested at a lateral pressure of $p=0.15$ $\mathrm{MPa}$ and an axial cyclic stress amplitude of $\sigma_{0}=0.10 \mathrm{MPa}$. Measured values of $\alpha$ and $\beta$ as functions of frequency are given in Table 4. Equations (23) and (28) have been used to tabulate the values of $J_{1}(\omega)$ and $J_{2}(\omega)$ in the same table. The conversion of this information to the time domain is described in the next section.

\section{Properties in Time Domain}

The complex compliances can be curve fit in the frequency domain by using equations of the following form:

$$
J_{1}^{\prime}(\omega)=J_{\infty}(t \rightarrow \infty)+\sum_{k=1}^{n} \frac{\omega^{2} J_{1}^{\prime k}}{\left[\omega^{2}+1 /\left(\tau^{k}\right)^{2}\right]}
$$

where $J_{\infty} \equiv J_{1}(t \rightarrow \infty)$ is the long time compliance in the time domain, which is equivalent to the real part of the complex shear

Table 2. Marshall Mix Aggregate Gradations

\begin{tabular}{lcccccccc}
\hline Marshall mix & $18.0 \mathrm{~mm}(\%)$ & $12.5 \mathrm{~mm}(\%)$ & $5.00 \mathrm{~mm}(\%)$ & $2.00 \mathrm{~mm}(\%)$ & $900 \mu \mathrm{m}(\%)$ & $400 \mu \mathrm{m}(\%)$ & $160 \mu \mathrm{m}(\%)$ & $71 \mu \mathrm{m}(\%)$ \\
\hline 900901 Design & 100 & 90.9 & 60.1 & 36.2 & 21.0 & 11.9 & 7.3 & 5.0 \\
900901 Bottom lift & 100 & 96.0 & 63.2 & 39.1 & 23.3 & 11.9 & 6.5 & 3.9 \\
900901 Top lift & 100 & 92.5 & 62.9 & 40.1 & 23.7 & 12.3 & 6.7 & 4.0 \\
\hline
\end{tabular}


Table 3. Superpave Mix Aggregate Gradations

\begin{tabular}{|c|c|c|c|c|c|c|c|c|c|c|c|}
\hline Superpave mixes & $\begin{array}{c}25.0 \mathrm{~mm} \\
(\%)\end{array}$ & $\begin{array}{c}19.0 \mathrm{~mm} \\
(\%)\end{array}$ & $\begin{array}{c}12.5 \mathrm{~mm} \\
(\%)\end{array}$ & $\begin{array}{c}9.5 \mathrm{~mm} \\
(\%)\end{array}$ & $\begin{array}{c}4.75 \mathrm{~mm} \\
(\%)\end{array}$ & $\begin{array}{c}2.36 \mathrm{~mm} \\
(\%)\end{array}$ & $\begin{array}{c}1.18 \mathrm{~mm} \\
(\%)\end{array}$ & $\begin{array}{c}600 \mu \mathrm{m} \\
(\%)\end{array}$ & $\begin{array}{c}300 \mu \mathrm{m} \\
(\%)\end{array}$ & $\begin{array}{c}150 \mu \mathrm{m} \\
(\%)\end{array}$ & $\begin{array}{c}75 \mu \mathrm{m} \\
(\%)\end{array}$ \\
\hline 900902 Design & 100 & 100 & 78.6 & 60.9 & 34.0 & 23.0 & 16.6 & 11.6 & 8.0 & 5.6 & 4.2 \\
\hline 900902 Top lift & 100 & 100 & 83.8 & 67.1 & 38.9 & 25.1 & 17.5 & 11.9 & 8.3 & 5.8 & 4.1 \\
\hline 900903 Design & 100 & 100 & 78.6 & 60.9 & 34.0 & 23.0 & 16.6 & 11.6 & 8.0 & 5.6 & 4.2 \\
\hline 900903 Bottom lift & 100 & 100 & 78.5 & 58.5 & 34.0 & 22.6 & 16.5 & 11.6 & 8.3 & 5.9 & 4.2 \\
\hline 900959 Bottom lift & 100 & 100 & 83.6 & 67.6 & 35.2 & 22.5 & 16.1 & 11.4 & 8.4 & 6.1 & 4.4 \\
\hline 900959 Top lift & 100 & 100 & 81.3 & 65.9 & 33.9 & 21.2 & 15.4 & 11.1 & 8.1 & 5.8 & 4.3 \\
\hline
\end{tabular}

compliance at zero frequency, and $J_{1}^{\prime}{ }^{k}, J_{\infty}$, and $\tau^{k}$ are material properties that can be obtained from a curve fitting scheme applied to the experimental creep data (Schapery 1984a, b; Tschoegl 1989). The range of summation $n$ will depend on the time-dependent nature of the material. For this analysis, the necessary value of $n$ was four.

The data shown in Table 4 have been curve fit using the technique referenced previously, and the properties for the four materials described herein are listed in Table 5. Figure 3 has been provided as an example as to how the curves for the shear storage compliance $J_{1}^{\prime}$ fit the experimental data in the frequency domain at $0.1,1,5$, and $10 \mathrm{~Hz}$.

The truly expedient outcome of this experimental procedure is that the range of frequencies can be swept out in a relatively short period of time $(1 / 2 \mathrm{~h}$ at one temperature), so that the experiment can be completed in a short time span. Furthermore, for linear viscoelastic materials there exists a mathematical identity for transforming from the frequency domain to the time domain, so that Equation (22), for example, can be used to obtain the shear creep compliance. This transformation is given by (Christensen 1982.

$$
J_{1}(t)=J_{1}(\infty)+\frac{2}{\pi} \int_{0}^{\infty} J_{1}^{\prime}(\omega) \sin \omega t d \omega
$$

Thus, long term creep compliance values can be obtained from relatively short-term tests, resulting in accelerated material characterization.
The substitution of Equation (29) into transformation integral Equation (30) will result in the following Prony series in the time domain. For this analysis, four Prony terms were used to characterize the creep compliances. Thus, it is assumed that the bulk creep compliance can be adequately modeled by

$$
J_{1}(t)=J_{1}(\infty)+\sum_{k=1}^{n} J_{1}^{k} e^{-t / \tau^{k}}
$$

The properties obtained in Table 5 serve also to describe the creep compliance in the time domain. These results are plotted in Figures 4 and 5. Experimental evidence for a wide range of linear viscoelastic media (Christensen 1982) suggests that a Prony series is both physically and mathematically appropriate for this purpose.

\section{Mathematical Principles for Obtaining Relaxation Moduli}

As pointed out in the previous section, it is more convenient from an experimental standpoint to perform material characterization tests in load control, thus resulting in the evaluation of creep compliances. Unfortunately, computational algorithms that use viscoelastic material properties are generally more convenient to construct when a strain formulation is used such as that described in constitutive Equation (3) (Allen et al. 1994; Zocher et al. 1997). Therefore, it is usually necessary

Table 4. Triaxial Compression Test Data

\begin{tabular}{lccccccccc}
\hline Specimens & $\omega(\mathrm{Hz})$ & $\beta(-)$ & $\alpha^{-1}(\mathrm{MPa})$ & $\alpha\left(\mathrm{MPa}^{-1}\right)$ & $\varepsilon_{11}(\mathrm{~mm} / \mathrm{mm})$ & $\sigma_{0}(\mathrm{MPa})$ & $p(\mathrm{MPa})$ & $J_{1}\left(\mathrm{MPa}^{-1}\right)$ & $J_{2}\left(\mathrm{MPa}^{-1}\right)$ \\
\hline Specimen 900901 & 0.1 & 0.34 & 500 & $2.00 \mathrm{E}-03$ & $2.00 \mathrm{E}-04$ & 0.100 & 0.150 & $3.22 \mathrm{E}-03$ & $5.04 \mathrm{E}-03$ \\
& 1 & 0.27 & 750 & $1.33 \mathrm{E}-03$ & $1.33 \mathrm{E}-04$ & 0.100 & 0.150 & $2.03 \mathrm{E}-03$ & $3.08 \mathrm{E}-03$ \\
& 5 & 0.15 & 1,000 & $1.00 \mathrm{E}-03$ & $1.00 \mathrm{E}-04$ & 0.100 & 0.150 & $1.38 \mathrm{E}-03$ & $1.95 \mathrm{E}-03$ \\
Specimen 900902 & 10 & 0.07 & 1,100 & $9.09 \mathrm{E}-04$ & $9.09 \mathrm{E}-05$ & 0.100 & 0.150 & $1.17 \mathrm{E}-03$ & $1.55 \mathrm{E}-03$ \\
& 0.1 & 0.28 & 500 & $2.00 \mathrm{E}-03$ & $2.00 \mathrm{E}-04$ & 0.100 & 0.150 & $3.07 \mathrm{E}-03$ & $4.68 \mathrm{E}-03$ \\
& 1 & 0.13 & 750 & $1.33 \mathrm{E}-03$ & $1.33 \mathrm{E}-04$ & 0.100 & 0.150 & $1.81 \mathrm{E}-03$ & $2.52 \mathrm{E}-03$ \\
Specimen 900903 & 5 & 0.05 & 850 & $1.18 \mathrm{E}-03$ & $1.18 \mathrm{E}-04$ & 0.100 & 0.150 & $1.48 \mathrm{E}-03$ & $1.94 \mathrm{E}-03$ \\
& 10 & 0.06 & 900 & $1.11 \mathrm{E}-03$ & $1.11 \mathrm{E}-04$ & 0.100 & 0.150 & $1.41 \mathrm{E}-03$ & $1.87 \mathrm{E}-03$ \\
Specimen 900959 & 0.1 & 0.34 & 550 & $1.82 \mathrm{E}-03$ & $1.82 \mathrm{E}-04$ & 0.100 & 0.150 & $2.92 \mathrm{E}-03$ & $4.582 \mathrm{E}-03$ \\
& 1 & 0.15 & 750 & $1.33 \mathrm{E}-03$ & $1.33 \mathrm{E}-04$ & 0.100 & 0.150 & $1.84 \mathrm{E}-03$ & $2.60 \mathrm{E}-03$ \\
& 10 & 0.1 & 900 & $1.11 \mathrm{E}-03$ & $1.11 \mathrm{E}-04$ & 0.100 & 0.150 & $1.47 \mathrm{E}-03$ & $2.00 \mathrm{E}-03$ \\
& 0.1 & 0.21 & 600 & $1.67 \mathrm{E}-03$ & $1.67 \mathrm{E}-04$ & 0.100 & 0.150 & $2.42 \mathrm{E}-03$ & $3.55 \mathrm{E}-03$ \\
& 1 & 0.11 & 750 & $1.33 \mathrm{E}-03$ & $1.33 \mathrm{E}-04$ & 0.100 & 0.150 & $1.78 \mathrm{E}-03$ & $2.44 \mathrm{E}-03$ \\
& 5 & 0.09 & 850 & $1.18 \mathrm{E}-03$ & $1.18 \mathrm{E}-04$ & 0.100 & 0.150 & $1.54 \mathrm{E}-03$ & $2.08 \mathrm{E}-03$ \\
& 10 & 0.08 & 900 & $1.11 \mathrm{E}-03$ & $1.11 \mathrm{E}-04$ & 0.100 & 0.150 & $1.44 \mathrm{E}-03$ & $1.93 \mathrm{E}-03$ \\
\hline
\end{tabular}


Table 5. Prony Terms for Asphalt Concrete Creep Compliances

\begin{tabular}{lrrrr}
\hline Specimens & $n(-)$ & \multicolumn{1}{c}{$J_{1 n}\left(\mathrm{MPa}^{-1}\right)$} & $J_{2 n}\left(\mathrm{MPa}^{-1}\right)$ & $\tau_{n}(\mathrm{~s})$ \\
\hline Specimen 900901 & $\infty$ & $3.50 \mathrm{E}-03$ & $5.30 \mathrm{E}-03$ & - \\
& 1 & $22.89 \mathrm{E}-04$ & $22.11 \mathrm{E}-04$ & 20.0 \\
& 2 & $21.31 \mathrm{E}-03$ & $22.28 \mathrm{E}-03$ & 2.0 \\
& 3 & $22.15 \mathrm{E}-04$ & $22.73 \mathrm{E}-04$ & 1.0 \\
Specimen 900902 & 4 & $26.57 \mathrm{E}-04$ & $21.23 \mathrm{E}-03$ & 0.2 \\
& $\infty$ & $3.50 \mathrm{E}-03$ & $5.30 \mathrm{E}-03$ & - \\
& 1 & $24.58 \mathrm{E}-04$ & $26.46 \mathrm{E}-04$ & 20.0 \\
& 2 & $21.65 \mathrm{E}-03$ & $22.68 \mathrm{E}-03$ & 2.0 \\
Specimen 900903 & 3 & $1.81 \mathrm{E}-04$ & $3.83 \mathrm{E}-05$ & 1.0 \\
& 4 & $22.06 \mathrm{E}-04$ & $21.84 \mathrm{E}-04$ & 0.2 \\
& 1 & $3.20 \mathrm{E}-03$ & $4.80 \mathrm{E}-03$ & - \\
& $22.73 \mathrm{E}-04$ & $21.35 \mathrm{E}-04$ & 20.0 \\
& 2 & $21.59 \mathrm{E}-03$ & $23.06 \mathrm{E}-03$ & 2.0 \\
Specimen 900959 & 3 & $4.21 \mathrm{E}-04$ & $8.31 \mathrm{E}-04$ & 1.0 \\
& 4 & $25.71 \mathrm{E}-04$ & $28.70 \mathrm{E}-04$ & 0.2 \\
& $\infty$ & $2.70 \mathrm{E}-03$ & $3.80 \mathrm{E}-03$ & - \\
& 1 & $23.11 \mathrm{E}-04$ & $22.39 \mathrm{E}-04$ & 20.0 \\
& 2 & $28.55 \mathrm{E}-04$ & $21.62 \mathrm{E}-03$ & 2.0 \\
& 3 & $1.65 \mathrm{E}-04$ & $3.86 \mathrm{E}-04$ & 1.0 \\
& 4 & $23.24 \mathrm{E}-04$ & $24.93 \mathrm{E}-04$ & 0.2 \\
\hline
\end{tabular}

to convert the experimentally determined creep compliances to relaxation moduli. This can be accomplished by implementing an additional step into the process of evaluating material properties. The compliances must be inverted to obtain the relaxation moduli shown in Equation (3). To see how this may be accomplished, consider the isotropic form of Equation (3) in deviatoric form, similar to Equations (14) and (15) as follows:

$$
\sigma_{k k}(t)=\int_{-\infty}^{t} G_{2}(t-\tau) \frac{\partial \varepsilon_{k k}}{\partial \tau} d \tau
$$

where $G_{2}(t)$ is called the bulk relaxation modulus, and

$$
\sigma_{i j}^{\prime}=\int_{-\infty}^{t} G_{1}(t-\tau) \frac{\partial \varepsilon_{i j}^{\prime}}{\partial \tau} d \tau
$$

where $G_{1}(t)$ is called the shear relaxation modulus. Although the relaxation moduli in principle can be obtained directly experimentally, experiments that are run in displacement control

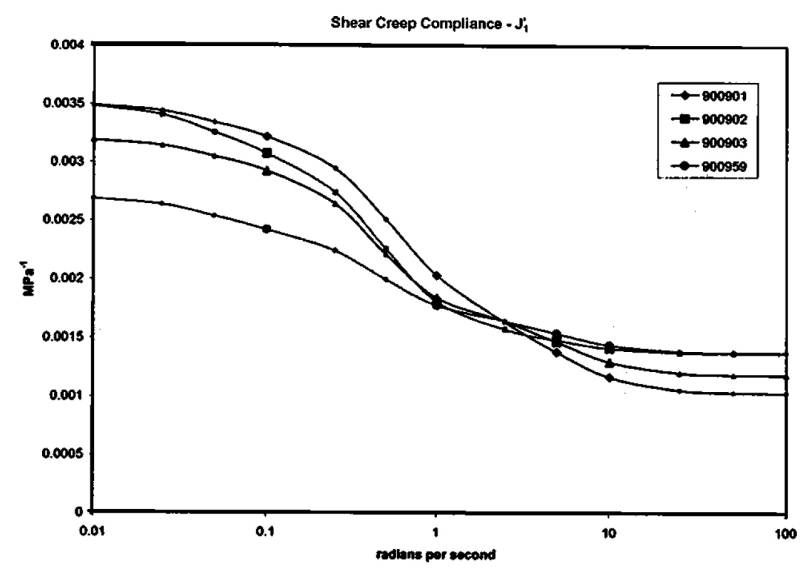

Figure 3. Shear storage compliances versus frequency for four asphalt concrete mixes

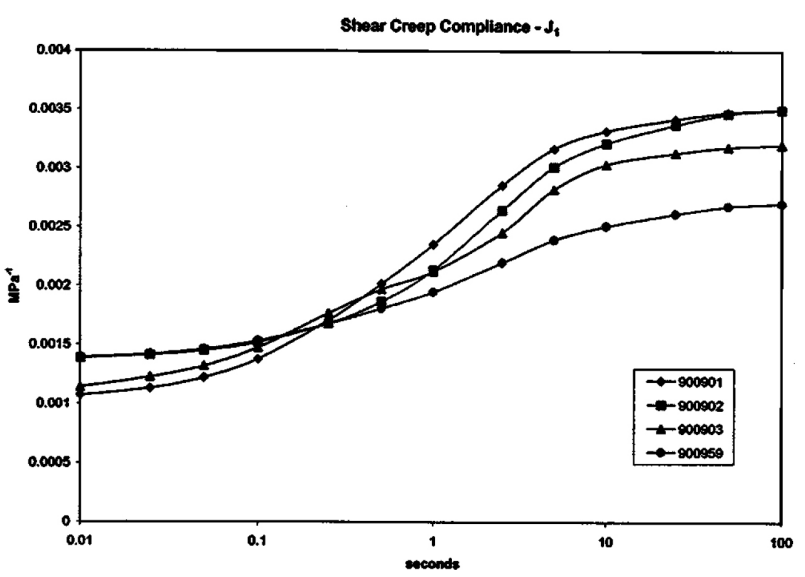

Figure 4. Shear creep compliances versus time for four asphalt concrete mixes

are somewhat more difficult to perform accurately, so that it is preferred to obtain the creep compliances from accelerated tests in the frequency domain by the method described previously and then derive the relaxation moduli from them. With this thought in mind, first consider hypothetically a creep test performed in shear such that from Equation (14)

$$
J_{1}(t)=\frac{\varepsilon_{12}(t)}{\sigma_{12}}
$$

Substitution of this result into Equation (33) will result in

$$
H(t)=\int_{-\infty}^{t} G_{1}(t-\tau) \frac{\partial J_{1}(\tau)}{\partial \tau} d \tau
$$

The previous provides a relationship between the shear relaxation modulus and shear creep compliance (a similar relationship may be obtained for the bulk properties). Unfortunately, the relation is in an implicit form that is not easily manipulated into a form that can be used to solve for $G_{1}(t)$ given $J_{1}(t)$. One possibility that presents itself is to Laplace transform (Greenburg 1978) Equation (35), thus resulting in

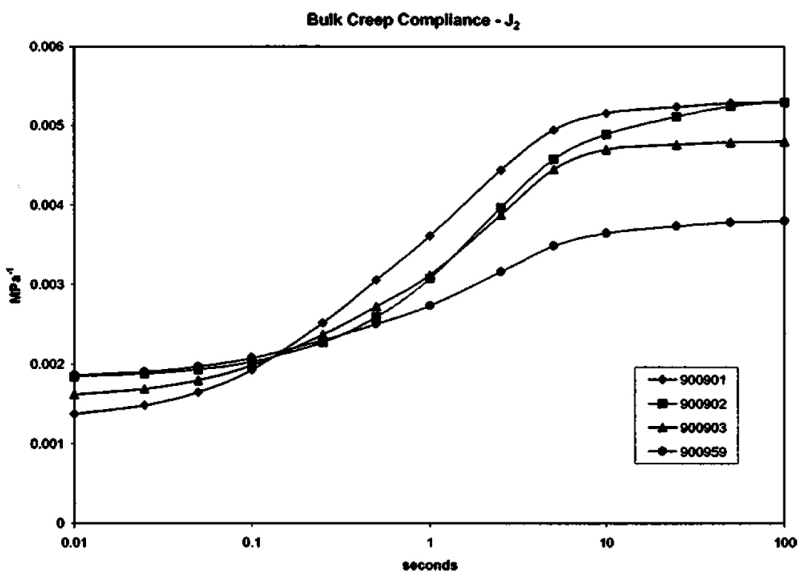

Figure 5. Bulk creep compliances versus time for four asphalt concrete mixes 


$$
\bar{G}_{1}(s)=\frac{1}{s^{2} \overline{J_{1}}(s)}
$$

where $s$ is the Laplace transform variable, and overbars signify the Laplace transform of a quantity. Equation (36) is potentially useful in obtaining the shear relaxation modulus. However, there are two shortcomings. First, it is necessary to Laplace transform the shear creep compliance $J_{1}(t)$. Laplace transforming Equation (31) results in

$$
\widetilde{J}_{1}(s)=J_{1}(t \rightarrow \infty)+\sum_{k=1}^{n} \frac{s J_{1}^{k}}{\left(s+1 / \tau^{k}\right)}
$$

where $\tilde{J}_{1}(s)$ is the s multiplied Laplace transform of $J_{1}(t)$ (called the Carson transform). Second, substituting Equation (37) into Equation (36) will result in an equation that can be inverse Laplace transformed in closed form for only small values of $n$.

When $n$ is large, it is preferred to use an inversion technique called the direct method (Schapery 1962). This method is used by substituting $0.56 / t$ directly for $s$ in the Laplace transformed equation as follows:

$$
G_{1}(t)=\left.s \bar{G}_{1}(s)\right|_{s \rightarrow 0.56 / t}
$$

The above technique has been shown to be exact when the function exhibits zero curvature in log-log space (Schapery 1962). Although it does not result in a Prony series for $G_{1}(t)$, it has been found that it is a simple matter to plot the resulting curve as a function of time, and then refit the result with a Prony series by the method described previously (Schapery 1984a,b; Tschoegl 1989). For this analysis, four Prony terms were used to characterize the relaxation moduli. The second author and coworkers have previously used this technique successfully on a glass mat thermoplastic (Megnis et al. 2001).

Using this technique results in a Prony series for the relaxation modulus that is of the form

$$
G_{1}(t)=G_{1}(0)+\sum_{k=1}^{n} G_{1}^{k} e^{-t / \tau_{k}}
$$

Table 6. Prony Terms for Asphalt Concrete Relaxation Moduli

\begin{tabular}{lrrrr}
\hline$m$ & $n(--)$ & \multicolumn{1}{c}{$G_{1 n}(\mathrm{MPa})$} & $\mathrm{G}_{2 n}(\mathrm{MPa})$ & $\tau_{n}(\mathrm{~s})$ \\
\hline Specimen 900901 & $\infty$ & $2.86 \mathrm{E}+02$ & $1.89 \mathrm{E}+02$ & - \\
& 1 & $4.32 \mathrm{E}+01$ & $2.23 \mathrm{E}+01$ & 20.0 \\
& 2 & $1.36 \mathrm{E}+02$ & $9.07 \mathrm{E}+01$ & 2.0 \\
& 3 & $1.32 \mathrm{E}+01$ & $1.40 \mathrm{E}+01$ & 1.0 \\
Specimen 900902 & 4 & $3.36 \mathrm{E}+02$ & $2.70 \mathrm{E}+02$ & 0.2 \\
& $\infty$ & $2.86 \mathrm{E}+02$ & $1.89 \mathrm{E}+02$ & - \\
& 1 & $5.90 \mathrm{E}+01$ & $3.92 \mathrm{E}+01$ & 20.0 \\
& 2 & $1.67 \mathrm{E}+02$ & $1.21 \mathrm{E}+02$ & 2.0 \\
Specimen 900903 & 3 & $21.73 \mathrm{E}+00$ & $9.98 \mathrm{E}+00$ & 1.0 \\
& 4 & $2.18 \mathrm{E}+02$ & $1.93 \mathrm{E}+02$ & 0.2 \\
& 1 & $3.13 \mathrm{E}+02$ & $2.08 \mathrm{E}+02$ & - \\
& 2 & $1.94 \mathrm{E}+01$ & $2.53 \mathrm{E}+01$ & 20.0 \\
Specimen 900959 & 3 & $23.18 \mathrm{E}+00$ & $1.14 \mathrm{E}+02$ & 2.0 \\
& 4 & $2.48 \mathrm{E}+02$ & $2.09 \mathrm{E}+02$ & 1.0 \\
& 1 & $3.70 \mathrm{E}+02$ & $2.63 \mathrm{E}+02$ & - \\
& 1 & $5.95 \mathrm{E}+01$ & $3.26 \mathrm{E}+01$ & 20.0 \\
& 2 & $1.36 \mathrm{E}+02$ & $1.04 \mathrm{E}+02$ & 2.0 \\
& 3 & $22.08 \mathrm{E}+01$ & $27.67 \mathrm{E}+00$ & 1.0 \\
& 4 & $1.57 \mathrm{E}+02$ & $1.30 \mathrm{E}+02$ & 0.2 \\
\hline
\end{tabular}

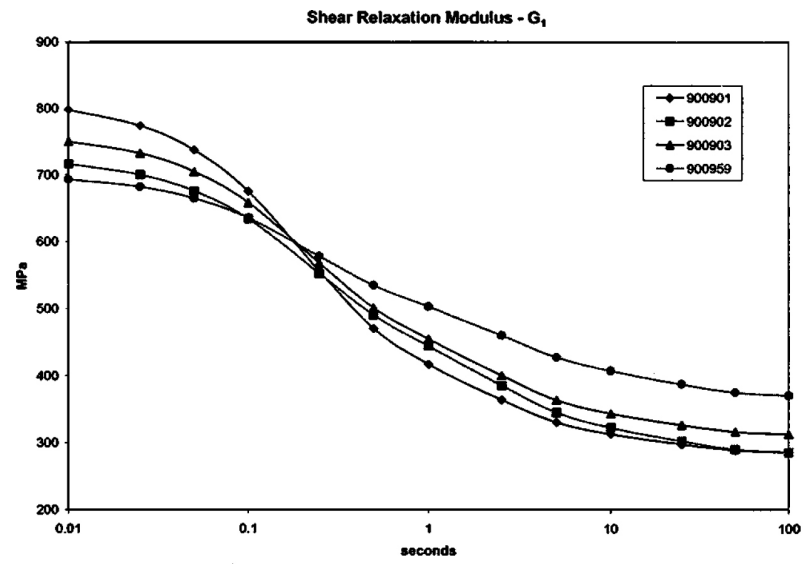

Figure 6. Shear relaxation moduli versus time for four asphalt concrete mixes

For the purpose of demonstrating this technique, triplicate samples of four asphalt mixes were characterized at room temperature and axially-sinusoidal stress levels. Using this technique results in the properties for the four asphalt mixes described in Table 6. For convenience, these results are also plotted in Figures 6 and 7.

\section{Conclusion}

A combined experimental and analytic method has been described for obtaining the linear viscoelastic properties of isotropic asphaltic pavement material. The technique has been used to produce complex compliances, creep compliances, and relaxation moduli for several different mixes of asphaltic pavement material. As described in the section entitled "Viscoelastic Material Model," once these properties are known the properties may be implemented to a continuum mechanics model to predict the response of roadways for a variety of loading conditions, material properties, and structural cross sections. Adjoined with appropriate fracture properties, these complex compliances, creep compliances, and relaxation moduli can be used to model pavement distresses such as thermal cracking, fatigue cracking, and rutting.

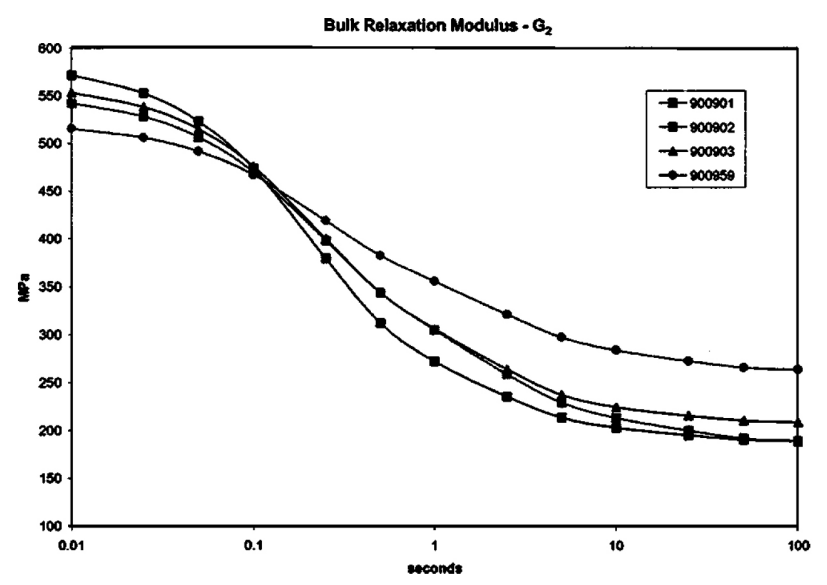

Figure 7. Bulk relaxation moduli versus time for four asphalt concrete mixes 
It is possible to use the resulting material properties to predict stresses, strains, and displacements in an asphaltic roadway for a variety of loading conditions, geometries, and material properties, and these results can then be used to predict roadway life as a function of these quantities. The necessity to perform full-scale roadway testing is thus obviated by this design approach. Therefore, the model described can be used as a design tool for improving roadway life at considerable cost savings to the public. Indeed, this process has been previously used by the second author in a variety of other structural applications (Foulk et al. 1998; Helms et al. 1999; Phillips et al. 1999; Allen et al. 2001a). The application of this design procedure to asphaltic roadways will be the subject of a future paper by the authors.

Finally, certain physical mechanisms can introduce nonlinearity into the viscoelastic response of the asphaltic concrete. One significant source of this nonlinearity is microcracking that can occur primarily within the binder, on a length scale that is normally commensurate with that of the aggregate particle size. The second author, together with other researchers (Helms et al. 1999), has studied this problem for particulate composites, and the application of this approach to asphaltic concrete will be the subject of future research by the authors.

\section{Acknowledgment}

The writers gratefully acknowledge the support provided for this research by the Saskatchewan Department of Highways and Transportation.

\section{References}

Allen, D. H., Holmberg, J. A., Ericson, M., Lans, L., Svensson, N., and Holmberg, S. (2001a). "Modeling of viscoelastic response of GMT structural components." Compos. Sci. Technol., 61, 503-515.

Allen, D. H., Jones, R. H., and Boyd, J. G. (1994). “Micromechanical analysis of a continuous fiber metal matrix composite including the effects of matrix viscoplasticity and evolving damage." J. Mech. Phys. Solids, 42(3), 505-529.

Allen, D. H., Searcy, C. R., and Berthelot, C. F. (2001b). “A micromechanical model for the accelerated characterization of viscoelastic constitutive behavior of asphaltic concrete." TEES Rep. No. CMC2001-1, Texas Engineering Experiment Station, College Station, Tex.

Allen, D. H., and Yoon, C. (1998). "Homogenization techniques for thermoviscoelastic solids containing cracks." Int. J. Solids Struct., 35, 4035-4054.

American Association of State Highway and Transportation Officials (AASHTO). (1995). "Standard specifications for transportation materials and methods of sampling and testing." 17th Ed., Washington, D.C.

ASTM. (1996). "Road and paving materials: Paving management technologies." Vol. 04.03, West Conshohocken, Pa.

Berthelot, C. F. (1999). Mechanistic modeling of Saskatchewan specific pavement studies-9A asphalt concrete pavement, $\mathrm{PhD}$ thesis, Texas A\&M Univ., College Station, Tex.

Christensen, R. M. (1982). Theory of viscoelasticity: An introduction, 2nd Ed., Academic, San Diego.
Foster, C. (1993). “Development of Marshall procedures for designing asphalt paving mixtures." National Asphalt Pavement Association, Rep., No. IS-84, Lanham, Md.

Foulk, III, J. W., Allen, D. H., and Helms, K. L. E. (1998). “A model for predicting the damage and environmental degradation dependent life of SCS-6/Timetal®21S [0]4 metal matrix composite." Mech. Mater., 29(1), 53-68.

Greenburg, M. D. (1978). Foundations of applied mathematics, Prentice- Hall, Englewood Cliffs, N.J.

Griffith, A. A. (1920). "The phenomena of rupture and flow of solids." Philos. Trans. R. Soc. London, A221, 163-197.

Helms, K. L. E., Allen, D. H., and Hurtado, L. D. (1999). “A model for predicting grain boundary cracking in polycrystalline viscoplastic materials including scale effects." Int. J. Fract., 95, 175-194.

Kim, J. R., Drescher, A., and Newcomb, D. E. (1997). “Rate sensitivity of asphalt concrete in triaxial compression." J. Mater. Civ. Eng., 9(2), 76-84.

Kim, Y. R., Lee, Y. C., and Lee, H. J. (1995). “Correspondence principle for characterization of asphalt concrete." J. Mater. Civ. Eng., 7(1), 59-68.

Kim, Y. R., and Little, D. N. (1990). “One-dimensional constitutive modeling of asphalt concrete." J. Eng. Mech., 116(4), 751-772.

Lee, H.-J., Daniel, J. S., and Kim, Y. R. (2000). “Continuum damage mechanics-based fatigue model of asphalt concrete." J. Mater. Civ. Eng., 12(2), 105-112.

Little, D., Button, J., and Youssef, H. (1992). “Development of criteria to evaluate uniaxial creep data and asphalt concrete permanent deformation potential." Transportation Research Record, No. 1417, Washington, D.C., 49-57.

Malvern, L. E. (1969). Introduction to the mechanics of a continuous medium, Prentice-Hall, Englewood Cliffs, N.J.

Matthews, J., and Monismith, C. (1994). "Investigation of laboratory fatigue testing procedures for asphalt aggregate mixtures," J. Transp. Eng., 119(4), 634 -654.

Megnis, M., Varna, J., Allen, D. H., and Holmberg, J. A. (2001).“A micro-mechanical modelling of viscoelastic response of GMT composite." J. Compos. Mater., 35, 849-882.

Oden, J. T. (1979). Applied functional analysis: A first course for students of mechanics and engineering science, Prentice-Hall, Englewood Cliffs, N.J.

Park, S. W., Kim, Y. R., and Schapery, R. A. (1996). “A viscoelastic continuum damage model and its application to uniaxial behavior of asphalt concrete." Mech. Mater., 24, 241-255.

Phillips, M. L., Yoon, C., and Allen, D. H. (1999). “A computational model for predicting damage evolution in laminated composite plates." J. Eng. Mater. Technol., 21, 436-444.

Schapery, R. A. (1962). "Approximate methods of transform inversion for viscoelastic stress analysis." Proc., 4th U.S. Congress Appl. Mech., ASME, New York, 1075-1086.

Schapery, R. A. (1981). “On viscoelastic deformation and failure behavior of composite materials with distributed flaws." Advances in Aerospace Structures and Materials, AD-01, 5-20.

Schapery, R. A. (1982). "Models for damage growth and fracture in nonlinear viscoelastic composites." Proc., 9th U.S. National Cong. of Applied Mechanics, H00228, ASME, New York, 237-245.

Schapery, R. A. (1984a). “Correspondence principles and a generalized J integral for large deformation and fracture analysis of viscoelastic media." Int. J. Fract., 25, 195-223. 
Schapery, R. A. (1984b). "Viscoelastic behavior and analysis of composite materials," Mechanics of composite materials, G. P. Sendeckyj, ed., Academic, San Diego, 85-168.

Schapery, R. A. (1987a). “Deformation and fracture characterization of inelastic composite materials using potentials." Polymer Engineering, 27, 63-76.

Schapery, R. A. (1987b). “Nonlinear constitutive equations for solid propellant based on a work potential and micromechanical model." Proc., 1987 JANNAF Struc. and Mech. Behavior Meeting, CPIA.

Schapery, R. A. (1990a). "Simplifications in the behavior of viscoelastic composites with growing damage." Proc., IUTAM Symp. on Inelastic Deformation of Composite Materials, Springer, New York, 193-214.

Schapery, R. A. (1990b). "Theory of mechanical behavior of elastic media with growing damage and other changes in structure." J. Mech. Phys. Solids, 38, 215-253.

Schapery, R. A. (1994). "Nonlinear viscoelastic constitutive equations for composites based on work potentials." Mechanics USA 1994 Proc. 12th U.S. Cong. Appl. Mech., Appl. Mech. Reviews, 47, 267-275.
Schapery, R. A., and Sicking, D. L. (1995). “On nonlinear constitutive equations for elastic and viscoelastic composites with growing damage." Mechanical behavior of materials, A. Bakker, ed., Delft Univ. Press, Delft, The Netherlands, 45-76.

Texas Department of Transportation. (1995). Manual of testing procedures, Austin, Tex.

Truesdell, C., and Noll, W. (1965). Handbuch der physik, S. Flugge, ed., Vol. 3, No. 3, Springer, Berlin.

Tschoegl, N. W. (1989). The phenomenological theory of linear viscoelastic behavior: An introduction, Springer, New York, Berlin.

White, T. (1985). "Marshall procedures for design and quality control of asphalt mixtures." Proc., Assoc. of Asphalt Paving Technologists, 54, St. Paul, Minn., 421-431.

Witczak, M., and Fonseca, O. (1996). "A revised predictive model for the dynamic (complex. modulus of asphaltic mixtures." Transportation Research Board, Washington, D.C.

Zocher, M. A., Allen, D. H., and Groves, S. E. (1997). "A three dimensional finite element formulation for thermoviscoelastic orthotropic media." Int. J. Numer. Methods Eng., 40, 2267-2288. 